International Tinnitus Journal. 2019;23(1):47-51.

\title{
Development and standardization of tinnitus handicap inventory in Nepali
}

Anuj kumar Neupane ${ }^{1 *}$ Anup Ghimire ${ }^{2}$

Bebek Bhattarai ${ }^{3}$ Prashanth Prabhu ${ }^{4}$

\begin{abstract}
Background: Tinnitus is a frequent but sparsely understood condition of human ear featuring unusual sensation of acoustic energy. A symptom specific inventory forassessment of tinnitus may aid in understanding the severity of the problem and its impact on individuals' life. Initially, English version of THI was the only existing assessment tool available in measuring the impact of tinnitus. Being a self-report inventory, it is vital that the individuals with tinnitus understand the language foradministering instrument. Therefore, the present study aimed at developing and validating self-administering Tinnitus Handicap Inventory (THI) in Nepali language.
\end{abstract}

Methods: Standard method of translation-back-translation was utilized where English version of THI was translated and customized by four professors with degree in Nepalilanguage. Later it was administered on 20 native literate Nepali speakers whose first language was Nepali for content validity. Therefore it was administered on 120 patients and total scores under different subscales were measured.

Results: Out of 120 individuals, $20 \%$ of them had severe, $20 \%$ had moderate, $26 \%$ of them had mild and $34 \%$ of them had slight tinnitus handicap scores. Nepali version of THI was found to have an alpha value of 0.92 . Across three subscales (emotional,functional and catastrophic), Nepali version of THI got the alpha scores of $0.91,0.91$ and 0.80 and Item-total correlation of 0.26-0.74, 0.057-0.90 and 0.54-0.90 respectively.

Conclusions: Present study confirmed that the THI-Nepali version has good internal consistency and reliability as compare to the original English version and otherestablished versions. Therefore, THI-Nepali version can be used as a robust, easy to use, reliable and valid tool in self-assessment of tinnitus handicap in Nepali speaking individuals with tinnitus in clinical as well as research setting. However, test-retest reliability of the developed questionnaire is essential.

Keywords: Psychological response; tinnitus; inventory; handicap; questionnaire; nepali.

${ }^{1}$ Department of Audiology and Speech Therapy, C.U. Shah Medical College and Hospitals, India

${ }^{2}$ Department of Audiology, All India Institute of Speech and Hearing, India

${ }^{3}$ Department of E.N.T, Institute of Medicine, Nepal

${ }^{4}$ Department of Audiology, All India Institute of Speech and Hearing, India

*Send correspondence to:

Anuj kumar Neupane

Department of Audiology and Speech Therapy, C.U Shah medical college and Hospitals, India. Phone:+918792482481; E-mail:anujkneupane@gmail.com

Paper submitted to the ITJ-EM (Editorial Manager System) on March 08, 2019; and Accepted on May 21, 2019 


\section{INTRODUCTION}

Tinnitus is a frequent but sparsely understood condition of human ear featuring unusual sensation of acoustic energy that may range from buzzing, hissing to ringing subtypes. It may have minute annoyance to detrimental effect on individuals' daily activities which may lead to anxiety, depression, sleep deprivation and poor concentration resulting impaired personal as well as professional lifestyle. Around $10.1 \%$ to $14.5 \%$ of adults are found to have complaint of tinnitus ${ }^{1}$ with higher prevalence in males than their female counterparts. Moreover, the problem is found to boost up with the increasing age. Even though the past decades were invested in understanding the rationale behind tinnitus and its possible etiologies, the lacunae regarding acute method of assessment and management of the condition still persists ${ }^{2}$. Studies have been performed to understand the severity of the tinnitus on individuals' life through various methods $^{3-5}$. As a result it was understood that the overall effect of tinnitus in individuals' life depends on its physical features as well as individuals' Psychological response to the problem. Hence it was recommended to use tinnitus reducing measures (e.g., tinnitus masker) so as to reduce the magnitude of tinnitus, whereas it was suggested to use questionnaire/inventory to solve the psychological impact of tinnitus on individuals' life. A symptom specific inventory for assessment of tinnitus may aid in understanding the severity of the problem and its impact on individuals' life. Therefore, in recent years, more focus has been given to the symptom based treatment strategy of tinnitus where it intends at precise assessment of the condition with reliable and valid handicap inventory. The self- evaluation section of the inventory provides the objective data regarding the severity of tinnitus and therefore, it helps in the assessment of the condition prior and after the treatment given4. In 1990, Kuk et al. ${ }^{5}$ came up with the Tinnitus Handicap Inventory (THI) that quantified the severity and impact of tinnitus on an individual's daily living. Later, Newman et al. (1996) ${ }^{6}$ developed a self-administering modified beta version THI that consisted of 25 questions under three sub-sections: emotional, functional and catastrophic. This version of the self-reporting inventory has been found to be reliable and valid with good internal consistency ${ }^{4}$. Initially, the English version of THI was the only existing assessment tool available in measuring the impact of tinnitus on individuals' life. Being a self-report inventory, it is vital that the individuals with tinnitus understand the language for administering THI. Therefore, THI has been translated and validated across various foreign languages around the globe ${ }^{4-8}$. However, Nepali language, being widely spoken in Nepal, India (Sikkim, West Bengal), Bhutan and Burma $^{9}$, has been observed to have no translated and validated version of THI yet. Nepali speaking population residing in South Asian developing countries, are found to have lower literacy rate and barely understand English. Large number of population depends on Nepali language for general communication which makes them impossible to utilise these inventories ${ }^{10}$. Inability of the multilingual audiological and otological professionals in accurate and reliable translation of the $\mathrm{THI}$ to Nepali language cannot be overlooked. Therefore, there is a need to develop and standardize the Tinnitus Handicap Inventory (THI) in Nepali language. The present study aimed at developing and validating the self-administering Tinnitus Handicap Inventory (THI) in Nepali language.

\section{MATERIALS AND METHODS}

\section{Development of the questionnaire}

In the present study, Tinnitus Handicap Inventory (THI) by Newman et al., (1996) ${ }^{6}$ was translated into Nepali language using the standard method of translationback-translation by four professors with degree in Nepali language. For content validity, the translated version of Tinnitus Handicap Inventory-Nepali (THI-Nepali version) was then administered on 20 native literate Nepali speakers whose first language was Nepali. The speakers were instructed to mark the questions on a Likert's scale of 5-point rating where 5 being 'not at all familiar' and 1 being 'very familiar'. The questions which were rated as 1 and 2 were adapted to the THI-Nepali version and questions which were rated above 2 were reframed and tested again for content validity. The final version of THINepali version consisted of questions which were rated as 1 or 2." It consists of 25 questions of THI-Nepali version in Nepali language and for easy understanding it is set in English translation in Appendix 1.

\section{Participants}

A total of 120 individuals with a mean age of 27.8 years and a standard deviation of 7.22 were selected for the study based on the inclusion and exclusion criteria. Among 120 individuals included, 60 males and 60 females between the age ranges of 18 to 44 suffering from tinnitus with or without any otological diseases were selected for the study. Individuals with complaint of tinnitus for more than 3 months were included for the study. None of these individuals reported any significant neurological history of psychiatric or psychological issues. All the individuals had a minimum qualification of SSLC (Secondary School Leaving Certificate) and were proficient in reading and writing Nepali language.

\section{Procedure}

Individuals were instructed to read the questionnaire thoroughly and complete the questionnaire comfortably in a given time on an individual basis. Following the administration of the tinnitus questionnaire, the individuals were instructed regarding the self -assessing THI-Nepali version, its importance and the system to grade it. Thereafter based on the subject's grading for all the questions, the total score was calculated. For each questions in THINepali version, 3 options were provided to the patient which they had to mark based on their perception of the 
problem namely YES, NO, and SOMETIMES. A score of four was given for YES, zero for $\mathrm{NO}$ and a score of two was given for SOMETIMES. Once the subject finished rating the questions, they were grouped under 5 categories based on the severity of tinnitus, ranging from Slight, Mild, Moderate, Severe and Catastrophic ${ }^{6}$.

\section{RESULTS}

After the detail analysis of the obtained data, the percentiles of the individuals across various severities were achieved. The total THI-Nepali version score of all the individuals ranged from 4 to 76 . This suggested that the severity of handicap ranged from slight to severe degree as represented in Figure 1. None of the individuals had catastrophic tinnitus. THI-Nepali version was able to categorize the tinnitus handicap as slight, mild, moderate and severe degree. The results of the study showed that out of 120 individuals, $20 \%$ of them had severe, $20 \%$ had a moderate, $26 \%$ of them had mild and $34 \%$ of them had slight tinnitus handicap scores. Also, the result of THI-Nepali version was analysed on the basis of three sub-sections: emotional, functional and catastrophic. In emotional subscale, YES, NO and SOMETIMES responses were found to be $12.5 \%, 52.5 \%$ and $35 \%$ of the times respectively. In case of functional subscale, $14.4 \%$ were YES, $56 \%$ were $\mathrm{NO}$ and $29.4 \%$ were SOMETIMES. For catastrophic subscale, $32 \%$ were YES, $52 \%$ were NO and $16 \%$ were SOMETIMES. The result of the study under

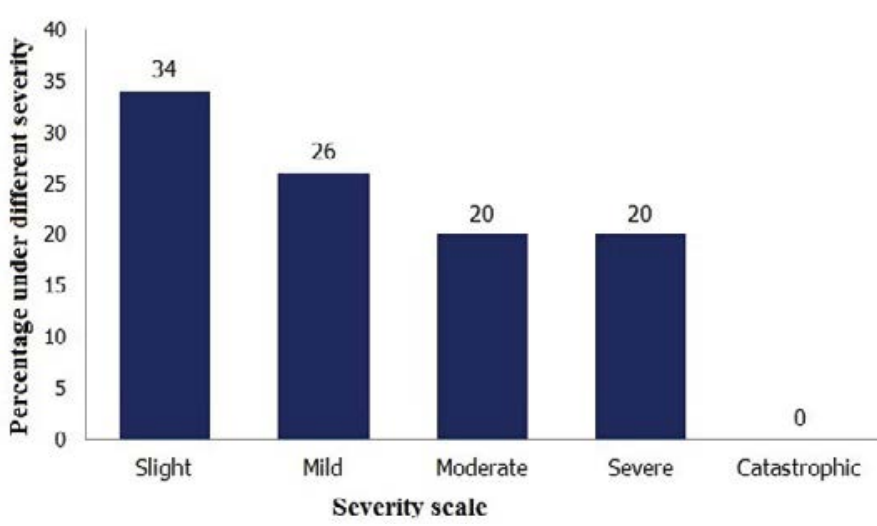

Figure 1. Percentage of individuals falling under different severities of tinnitus as per Tinnitus Handicap Inventory classification.

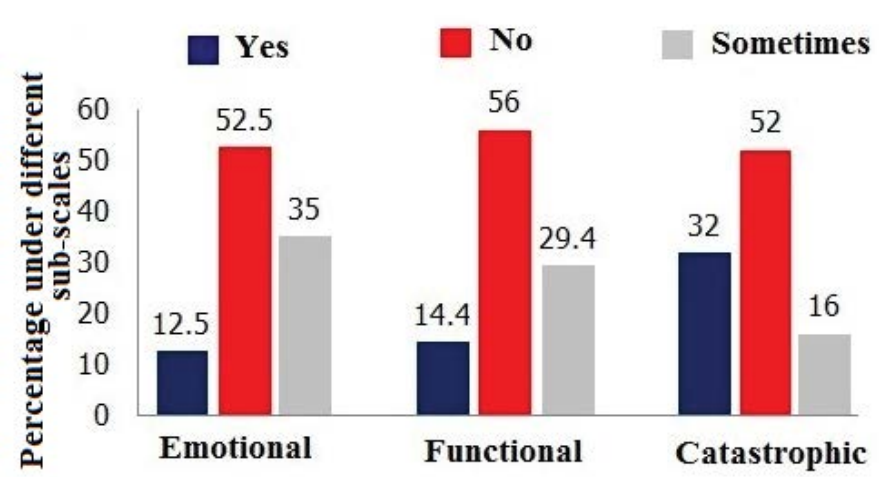

Figure 2. Percentage of individuals falling under different subscale of Tinnitus Handicap Inventory- Nepali (THI-N).

Table 1. Tinnitus Handicap Inventory- Nepali version (THI-N) with item - total correlation and Cronbach's Alpha values.

\begin{tabular}{|c|c|c|c|}
\hline SI. no & Items & $\begin{array}{l}\text { Item-total } \\
\text { correlation }\end{array}$ & $\begin{array}{c}\text { Cronbach's } \\
\text { Alpha if } \\
\text { item deleted }\end{array}$ \\
\hline $1 \mathrm{~F}$ & Due to the tinnitus, is it hard for you to concentrate? & 0.057 & 0.92 \\
\hline $2 \mathrm{~F}$ & Does the loudness of the tinnitus make it uncomfortable in listening? & 0.103 & 0.923 \\
\hline $3 \mathrm{E}$ & Does the tinnitus make you angry? & 0.255 & 0.919 \\
\hline $4 \mathrm{~F}$ & Are you confused due to the tinnitus? & 0.114 & 0.921 \\
\hline $5 \mathrm{C}$ & Do you feel desperate due to the tinnitus? & 0.754 & 0.909 \\
\hline $6 \mathrm{E}$ & Do you complain a great deal about the tinnitus? & 0.512 & 0.914 \\
\hline $7 \mathrm{~F}$ & Due to the tinnitus, do you have difficulty falling asleep at night? & 0.677 & 0.912 \\
\hline $8 \mathrm{C}$ & Have you ever felt that you cannot getaway the tinnitus? & 0.545 & 0.914 \\
\hline $9 \mathrm{~F}$ & Does the tinnitus hinder your social activities? & 0.543 & 0.914 \\
\hline $10 \mathrm{E}$ & Due to the tinnitus, do you feel frustrated? & 0.571 & 0.913 \\
\hline $11 \mathrm{C}$ & Do you feel that you have a terrible disease and so you are hearing the tinnitus? & 0.539 & 0.914 \\
\hline $12 \mathrm{~F}$ & Does the tinnitus hinder you to enjoy life? & 0.581 & 0.913 \\
\hline $13 \mathrm{~F}$ & Does the tinnitus hamper with your job performance or household responsibilities? & 0.553 & 0.915 \\
\hline $14 \mathrm{~F}$ & Due to the tinnitus, do you find that you are often irritable? & 0.701 & 0.911 \\
\hline $15 \mathrm{~F}$ & Due to the tinnitus, is it difficult for you to read? & 0.377 & 0.916 \\
\hline $16 \mathrm{E}$ & Does the tinnitus make you upset? & 0.64 & 0.912 \\
\hline $17 \mathrm{E}$ & Do you feel that the relationship with family and friends has been affected due to the stress on you by the tinnitus? & 0.48 & 0.915 \\
\hline $18 \mathrm{~F}$ & Do you find it hard to focus your attention away from the tinnitus and concentrate on other topics? & 0.904 & 0.907 \\
\hline $19 \mathrm{C}$ & Do you feel that you have no control over the tinnitus? & 0.484 & 0.915 \\
\hline $20 \mathrm{~F}$ & Do you often feel tired due to the tinnitus? & 0.45 & 0.916 \\
\hline $21 \mathrm{E}$ & Do you feel depressed due to the tinnitus? & 0.74 & 0.911 \\
\hline 22E & Does the tinnitus make you anxious? & 0.616 & 0.913 \\
\hline $23 C$ & Do you feel that you can no longer cope with the tinnitus? & 0.701 & 0.91 \\
\hline $24 \mathrm{~F}$ & Does the tinnitus get worse when you are under stress? & 0.793 & 0.909 \\
\hline $25 \mathrm{E}$ & Does the tinnitus make you feel insecure? & 0.725 & 0.91 \\
\hline
\end{tabular}


three sub-sections: emotional, functional and catastrophic is represented in Figure 2. Statistical package for social sciences (SPSS) version 21 was utilized to understand the internal consistency of the questionnaire with Cronbach's alpha. The result indicated Nepali version of THI with an alpha value of 0.92 suggesting good internal consistency. Also, the item-total correlation for THI-Nepali version was studied. Table 1 represents the Nepali version of Tinnitus Handicap Inventory (THI-N) with item - total correlation and Cronbach Alpha score for each 25 questions. Also, the overall Cronbach alpha score and individual scores for all the three subscales of THI-Nepali version were compared with THI-English version.

\section{DISCUSSION}

Tinnitus is a common symptom of otological and neurological conditions, adversely affecting social, professional and emotional aspects of life. Therefore, the symptom specific inventory for the assessment of tinnitus might help in understanding the severity of the problem and its impact on individuals' life. Moreover, selfadministering inventory provides insight regarding the severity of the problem, therefore, assisting in evaluation and possible intervention of the same 4 . Hence, the present study was conducted to develop and standardize the self-administering tinnitus handicap inventory in Nepali language. Present study revealed THI-Nepali version to be useful in quantifying various degrees of tinnitus in individuals' speaking Nepali language. Therefore, it might help in counseling individuals regarding the nature of the problem and possible strategy to cope up with ${ }^{2}$. Also, one could observe higher range of handicap scores across various questions in the study which might be due to the variation in the severity of the problem across individuals. This could be due to the variation among individuals' endurance to the problem and also coping mechanism adopted by each individual participated in the study ${ }^{6}$. In the present study, Cronbach's alpha score of 0.92 with the range of $0.057-0.904$ item-total correlation score was found. The Cronbach alpha value for the original THIEnglish version ${ }^{6}$ was 0.93 with an item total correlation of 0.22 to 0.77 . Similarly, the alpha score in Nepali was comparatively closer with THI developed in other languages such as Portuguese ${ }^{11}(\alpha=0.94)$, Turkish ${ }^{4}(\alpha=0.886)$, Italian ${ }^{12}$ $(\alpha=0.91)$ and Hebrew ${ }^{13}(\alpha=0.93)$. Present study also tried to understand the effect of tinnitus under three subscales: emotional, functional and catastrophic. Attempt was made to understand the effect of tinnitus in manifestation of anger, frustration and depression with emotional scale, effect of tinnitus in concentration, hearing acuity, attention \& reading with functional scale and effect of tinnitus in manifestation of negative thoughts such as intolerance, loss of control over the phenomenon and desperacy with catastrophic scale. While comparing the Cronbach alpha scores and item-total correlation across these subscales of emotional, functional and catastrophic, Nepali version of THI got the alpha scores of $0.91,0.91$ and 0.80 and Item- total correlation of $0.26-0.74,0.057-0.90$ and $0.54-0.90$ respectively. It was comparatively closer to the original English version of $\mathrm{THI}$ with alpha scores of $0.87,0.86$, and 0.68 and an Item-total correlation of $0.56-0.82,0.27-0.76$, and $0.42-0.48$ respectively across emotional, functional and catastrophic subscales respectively ${ }^{6}$. Similarly, the Turkish $\mathrm{THI}$ also had reported alpha values of $0.78,0.79$ and 0.68 across emotional, functional and catastrophic scales respectively ${ }^{4}$. The alpha values obtained in the present study were similar to those with the original THIEnglish version and other languages which was suggestive of good reliability and internal consistency of THI-Nepali version.

\section{Construct validity}

As there is no other self-administering tinnitus related handicap inventory in Nepali language, construct validity was obtained by correlating total score of THI-Nepali version with total score of each subscale (emotional, functional and catastrophic). Significant correlation was observed $(p=0.000)$ across the scores which revealed the consistency of the subscales in measuring the aspects it is intended to.

\section{CONCLUSION}

Most region of South Asia with Nepali population follows English version of $\mathrm{THI}$ in audiological and otorhinolaryngological settings to administer the degree of tinnitus. This is found to be difficult for native Nepali speakers for whom even official language is Nepali. Hence, the present study focused on the development and standardization of THI-Nepali version, and therefore selfadminister on Nepali speaking individuals with tinnitus. Present study confirmed that the THI-Nepali version has good internal consistency and reliability as compare to the original English version and other established versions. This version of $\mathrm{THI}$ will help individuals with tinnitus to understand the severity of their problem as well as its effect in their life. Therefore, THI-Nepali version can be used as a robust, easy to use, reliable and valid tool in self-assessment of tinnitus handicap in Nepali speaking individuals with tinnitus in clinical as well as research setting. However, test-retest reliability of the developed questionnaire is essential.

\section{CONFLICT OF INTEREST}

The Author declares no potential conflict of interest on publishing this paper.

\section{REFERENCES}

1. Davis A, El Refaie A. Epidemiology of tinnitus. Tinnitus handbook (Singular Audiology Text), Singular Pub. Group. 2000.

2. Biswas A. An Introduction to Neurotology. Mumbai: Bhalani House Publishing. 2006:293-98.

3. Dauman R, Tyler RS, Aran JM. Intracochlear electrical tinnitus reduction. Acta oto-laryngologica. 1993;113(3):291-5.

4. Aksoy S, Firat Y, Alpar R. The Tinnitus Handicap Inventory: a study of validity and reliability. Int Tinnitus J. 2007;13(2):94-8 
5. Kuk FK, Tyler RS, Russell D, Jordan H. The psychometric properties of a tinnitus handicap questionnaire. Ear Hear. 1990;11(6):434-45.

6. Newman CW, Jacobson GP, Spitzer JB. Development of the Tinnitus Handicap Inventory. Arch Otolaryngol Head Neck Surg. 1996;122(2):143-8.

7. Zacharia T, Naik PV, Sada S, Kuniyil JG, Dwarakanath VM. Development and standardization of tinnitus handicap inventory in Kannada. Int Tinnitus J. 2012; 17(2):117-23.

8. Aithal VU, Pillai VV, Zacharia T, Rajashekhar B. Development and standardization of tinnitus handicap inventory in malayalam. Int Tinnitus J. 2013;18(2):129-133.

9. Michailovsky B. Digitized resources for languages of Nepal. TRENDS Linguist Stud Monogr. 2006;175:243-4.
10. Eide AH, Neupane S, Hem K. Living conditions among people with disability in Nepal. SINTEF Rapport. 2016.

11. Schmidt LP, Teixeira VN, Dalligna C, Dallagnol D, Smith MM. Brazilian Portuguese Language version of the "Tinnitus Handicap Inventory": validity and reproducibility. Braz J Otorhinolaryngol. 2006;72(6):808-10.

12. Monzani D, Genovese E, Marrara A, Gherpelli C, Pingani L, Forghieri $\mathrm{M}$, et al. Validity of the Italian adaptation of the Tinnitus Handicap Inventory; focus on quality of life and psychological distress in tinnitus-sufferers. Acta Otorhinolaryngol Ital. 2008;28(3):126-34.

13. Oron $\mathrm{Y}$, Shushan S, Kreitler S, Roth $\mathrm{Y}$. A Hebrew adaptation of the tinnitus handicap inventory. Int J Audiol. 2011;50:426-30. 